\title{
Research on Regional Water Resources Development Threshold Model based on Rough Sets and the Support Vector Machine
}

\author{
Wang Jianjun
}

School of Civil Engineering, Shandong University; Ji'nan 250100, China.

\begin{abstract}
Keywords: Rough Sets, Support Vector Machine, Threshold Model, Water Resources, Regional.
\end{abstract}
\begin{abstract}
In this manuscript, we conduct research on regional water resources development threshold model based on rough sets and support vector machine. Through the analysis of the coupling system of economic and social interaction between water resources and sustainable development mode for the coupling system, multiple evaluation indexes by using fuzzy optimization model was transformed into a single index, fuzzy membership degree to describe the sustainable degree to measure complex water resources system sustainable development level of evaluation method. In addition, we combine the rough sets and support vector machine to enhance the traditional approaches on the corresponding issues that optimize and modify the performance of the threshold model.
\end{abstract}

\section{Introduction}

Along with the social economic development and industrial structure adjustment, in the process of development and utilization of the water resources supply and use, platoon, significant changes in the relationship between water consumption and water use structure, the structure change of increasing water consumption and water to be increasingly prominent contradiction between supply and demand of water resources, the urgent need for the development and utilization of water resources in the new situation and new problems in a timely manner to make the evaluation, and on the basis of planning, optimizing the allocation of water resources, the overall arrangement of life with production and the ecological water use, in order to meet the needs of water resources management in the new period, to the sustainable utilization of general water resources to support the sustainable development of social economy. Review past city water resources utilization can be found with the current scientific view of the development and environmental protection concepts do not fit the many problems: such as urban river water upstream the excessive development of basic urban area and the downstream river water shortage, water quality deterioration and even stop [1-2].

In order to effectively development and utilization of the water resources, China has built a large number of the water conservancy projects. The water conservancy project in the rational development and utilization of water resources and has played a great role in inundation and drought resistance also provides conditions for the sustainable development of our country. Although our country has made great achievements in the development and utilization of the water resources, but there are also many problems that could be summarized as follows. (1) Water pollution has become increasingly serious, the available water decreasing. Due to the low effluents in our country, most directly or indirectly into water bodies without treatment, water pollution is very outstanding. (2) Water resources are relatively abundant, but the lower level of the development and utilization of waste is serious. At present, the development and utilization of water resources is not high, the repeat utilization of water resources is low. (3) The spatial distribution of water resources and land resources distribution, the contradiction between supply and demand. This combination of the water and soil resources is not balanced, often overload and unreasonable utilization of soil resources, the formation of cultivated land, grassland, rivers problems such as the general land, thereby increasing floods caused the ecological environment deterioration, of course that will cause the regional differences of the national economy.

In this manuscript, we conduct research on regional water resources development threshold model based on rough sets and support vector machine. The tense situation intensifies as has become water security restriction factor for sustainable development of social economy in our country. Therefore, 
study water resources bearing capacity theory has very important and urgent practical significance. In the following sections, we will introduce our methodology in detail.

\section{The Proposed Methodology}

\subsection{Water Resources Development Principles.}

Water resources security is based on the stable status and functions of water resources system and improves the logo. State stability is water resources system internal structure complete as can keep the continuous and stability of the water cycle system. Functioning is water has the functions of normal work, the performance of the system is maintained, resist the change and interference of system as well as the ability to repair itself can maintain basic.

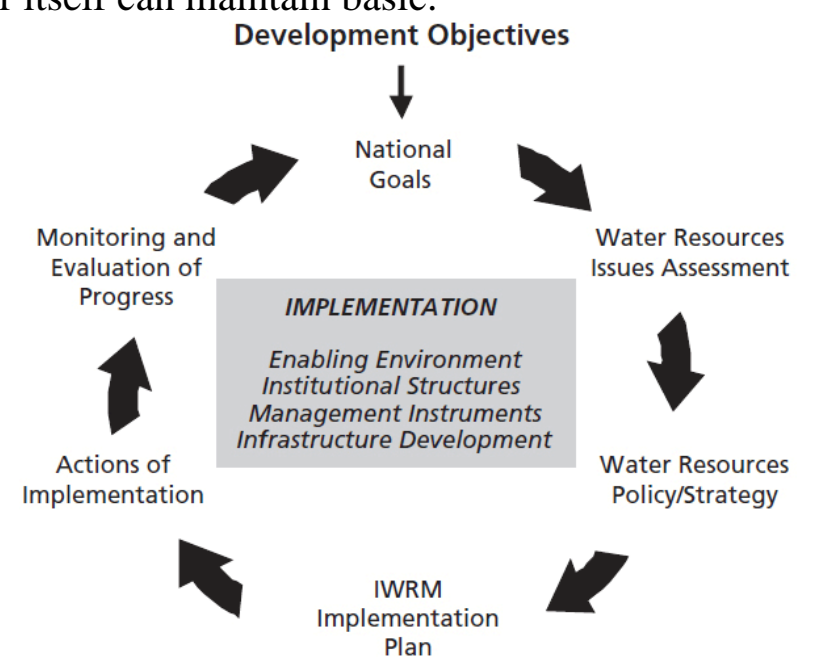

Fig. 1 The Water Resources Development Principles

From the perspective of system engineering, the water resources development and utilization is the complicated system engineering, its research object is a dynamic open complex giant system. Water resource is involved in a complex system, with the environment, economy, society, development and utilization of water resources will produce the corresponding benefit, also may bring adverse impact. To understand the actual level of a regional water resources while carrying the capacity, to judge its development to a certain stage can support the sustainable development of the economy, needs to be based on the theory of sustainable development and resource carrying from different space and time scale, analysis of water resources carrying condition and development trend [3].

The characteristics of the water resources development and utilization of complex giant system can be summarized as the follows. (1) Openness of system. In the process of development and utilization of water resources, from beginning to end is the participation of the people, by the outside world at the same time social system, economic system, the influence of the environmental system. (2) Complex correlation between subsystems. Interaction between each subsystem, connect with each other to form large system of the water resources development. (3) The diversity of the target. The ultimate goal of development and utilization of water resources and is to establish the starting point of development and utilization of water resources system, and for water resources evaluation standard.

\subsection{The Rough Sets.}

Rough set theory to deal with the data of the main process is: through the existing knowledge of database, data through a filling and general data preprocessing, attribute reduction and attribute value reduction and rule extraction, and a series of data operation, produce the new classification rules. By analyzing the generated rules can be concluded that the relationship between data and to extract useful information, to reduce the dimension of features and identify new information of intellectual property.

Rough set theory can deal with incomplete data, and under the certain error criterion processing incompatible data. In the process of the processing of these data, in addition to the problems to data 
collection, rough set theory does not need other additional prior information is based on the analysis of data, it is concluded that knowledge that is demonstrated in the figure 2.

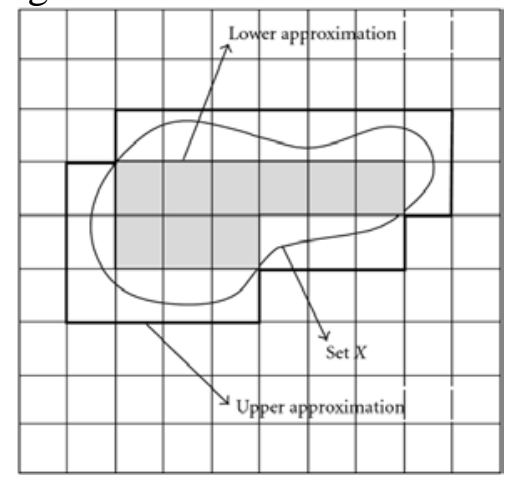

Fig. 2 The Demonstration of the Common Rough Set Pattern

In rough set theory, knowledge reduction and its algorithm is the key to the realization of decision table information processing. Knowledge roughness is, however, by the equivalence relation algebra and collection contains relationship to define, the definition is not easy to make people understand the nature of knowledge roughness that could be expressed as the follows.

$$
R_{-}(X)=\left\{X \in u:[x]_{R} \mid X \neq \Phi\right\}
$$

Reduction is defined as including redundant attributes and ensures correct classification of the minimum condition attribute set. A decision table may existed several reductions at the same time, the intersection is defined as the reduction of decision table nucleus and nucleus is one of the important properties affect the classification of attributes as the formula two.

$$
P(X)=\left\{x \in U \mid[x]_{P} \subseteq x\right\}
$$

\subsection{Support Vector Machine.}

Support vector machine was originally used to solve the problem of two kinds of classification, how to effectively promote it to more class classification problem or a basic problem that is working. Currently, there are many algorithms will support vector machine are generalized to the multiple class classification problem which is reflected from the figure three.

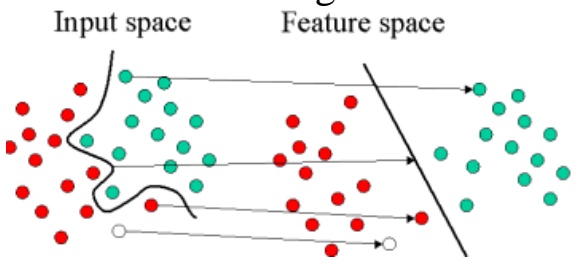

Fig. 3 The Procedures and Projection of the Support Vector Machine

Although the SVM method can give the model of the generalization of the strict boundary, thus ensuring the unknown sample produce correct decision results, but that does not eliminate the user preference for understandability. The modelling of the SVM can be expressed as follows.

$$
\min _{a} \frac{1}{2} a^{T} Q a-\exp (T) a
$$

Cross validation is common used a method of SVM parameters selection and easy to implement, the disadvantage is that large amount of the calculation, especially for large sample problem. The Bayesian method needs certain parameters of the prior knowledge of the space, and each time to determine the optimal parameters need multiple iterations, that compared with the cross validation method, on the amount of calculation and computational complexity advantage is not big, it is not easy to implement. Therefore, we should revise the condition as the follows [4].

$$
\max \sum_{i=1}^{n} a_{i}-\frac{1}{2} \sum_{i=1}^{n} \sum_{j=1}^{n} a_{i} a_{j}\left(x_{i} \times x_{j}\right)
$$

Compared with other types of kernel function, Gaussian RBF kernel is simple in model selection, calculation difficulty is small, the advantages of high calculation efficiency and the algorithm is easy 
to implement. Therefore, in the process of actual use tend to choose the Gaussian radial basis kernel function. Series distribution estimation algorithm only through the selection operator and gene pool to carry out reorganization operator evolution, the lack of mutation operator to maintain the population diversity, Therefore, introducing chaos mutation in the traditional SVM as an improved distribution estimation algorithm and the corresponding challenges.

\subsection{The Enhanced Threshold Model.}

The sustainability of human society, including ecological sustainability, economic sustainability and social sustainability as water resource is an important part of ecological and economic system, to obey the law of ecological and economic dominance, and for the ecological environment and social and economic development services and the existence of the sustainability of water resources, mainly by the natural water cycle of ecological sustainability principles and decisions.

Known from the analysis of the above, the threshold refers to the development of water resources throughout the development process of water resources, water resources system ultimately reached the maximum water supply capacity, that is to say, in the social production conditions, the conditions of economic and technical level have reached a level in the water resource for the industrial and the agricultural production system and the water capacity of people's living and ecological environment, namely the maximum capacity of the development of water resources, in this capacity, the water can natural circulation, updating, and constantly to use, and not cause the deterioration of the ecological environment at the same time and we update the traditional model as the formula 5.

$$
\mathrm{K}_{\text {enhanced }}=\min \left\{1, \sum_{i=1}^{m} a_{i} \otimes x_{i j}\right\}
$$

Water is not only the constitute elements of ecological structure, and also with the surrounding environment of frequent exchange of matter and the energy, so as to constitute a system function, the participation system of the material circulation and energy transformation to promote the evolution of the system that will enhance and optimize the corresponding industries.

\section{Conclusion and Summary}

In this paper, we conduct research on regional water resources development threshold model based on rough sets and the support vector machine. Water security situation is not only related to water resources condition and load condition, and loading status of the water environment, water ecological safety and security of water supply for the economic and social conditions. Comprehensive evaluation of water resources security is in the status quo of water resources sustainable utilization evaluation, loading status of water environment evaluation and water ecological security evaluation, and water supply security situation evaluation, on the basis of in water level area as a unit, in the process of the comprehensive evaluation of water resources security situation in the country. Under this background, we propose the related model to update the threshold pattern that is meaningful.

\section{References}

[1]. Zhao, $\mathrm{Xu}$, et al. "Physical and virtual water transfers for regional water stress alleviation in China." Proceedings of the National Academy of Sciences 112.4 (2015): 1031-1035.

[2]. Burns, Erick R., et al. "Evaluating geothermal and hydrogeologic controls on regional groundwater temperature distribution." Water Resources Research (2016).

[3]. Hering, Daniel, et al. "Managing aquatic ecosystems and water resources under multiple stress-An introduction to the MARS project." Science of the Total Environment 503 (2015).

[4]. Wang, Zhanqi, et al. "Optimal water resources allocation under the constraint of land use in the Heihe River Basin of China." Sustainability 7.2 (2015): 1558-1575. 